International Journal of Computer Science \& Information Technology (IJCSIT), Vol 3, No 1, Feb 2011

\title{
Characterization of tumor region using SOM and Neuro Fuzzy techniques in Digital Mammography
}

\author{
Anamika Ahirwar ${ }^{1}$, R.S. Jadon ${ }^{2}$ \\ ${ }^{1}$ Department of Computer Application, Madhav Institute of Technology \& Science, \\ Gwalior \\ E-mail: aanamika77@gmail.com \\ ${ }^{2}$ Department of Computer Application, Madhav Institute of Technology \& Science, \\ Gwalior \\ E-mail:rsj_mits@yahoo.com
}

\begin{abstract}
Nowadays the most common type of cancer in women is breast cancer. This is the second main cause of cancer deaths in women. Digital mammography is the technique which is used to examine the breast. This is very much useful for the detection of breast diseases in women. The automatic detection of tumor or some type of deformity in the medical imaging is done by many researchers to develop some algorithms and methods. In this paper we are using SOM and Fuzzy c-means clustering techniques for tumor detection in digital mammography images. We then further calculate the statistical features of tumor like location of tumor, area, energy, entropy, idm, mean, contrast, mean and standard deviation which helps the radiologist to study the statistical information regarding breast cancer, so that the doctors can give better treatment to patients. For calculating these statistical properties we use region growing and region merging techniques.
\end{abstract}

\section{Keywords}

Gray level cooccurrence matrix (GLCM), Best Matching Unit (BMU), epoch number, idm (inverse difference moment), micro-calcifications.

\section{Introduction}

Breast cancer has been one of the major causes of death among women since the last decades. This disease became a commonest cancer among women. If the cancer can be detected early, the options of treatment and the chances of total recovery will increase. Several efforts have been devoted to the improvement of breast cancer detection in the population. For example, via screening campaigns, breast self-examination orientation, clinical examinations, etc. The results of several years of research indicate that mammography is the main test used for screening and early diagnosis of breast cancer [23]. Small clusters of micro-calcifications [5,7,11,12] appearing as collection of white spots on mammograms show an early warning of breast cancer. A mammogram is 85-90 percent accurate. Therefore, 10-15 percent of cancers cannot be seen by mammogram. Some women are anxious about the consequences of radiation from a mammogram. Nowadays, the amount of radiation received is less for a mammogram than a chest X-ray. If in the early stage the cancer is detected, the death rate can be reduced. Radiologist takes the mammogram readings, so that the mammographers visually examine the

DOI : $10.5121 /$ ijcsit.2011.3114 
International Journal of Computer Science \& Information Technology (IJCSIT), Vol 3, No 1, Feb 2011

presence of deformities. And these deformities can infer as cancerous changes. Due to human errors which are caused by visual fatigue, the manual readings may consequence misdiagnosis. Computer aided diagnosis techniques are bring into play to improve the diagnostic accuracy and efficacy of screening mammography. In this work, we propose a clustering based hybrid technique combining the advantages of SOM (Self Organizing Map) and FCM (Fuzzy Cmeans), implemented for digital mammogram image segmentation process to detect various properties of image and tumor in digital mammography to find the deformity in the medical images. SOM or FCM separately as one of the tool for the image segmentation for tumor analysis and finding the cancer in mammography images. SOM and FCM are the image processing and image analysis algorithms. The benefit of using SOM is that it is very simple, very easy to understand and they work very well. As SOM classifies the data well and are easy to evaluate their own quality, so that we can calculate a good map and the strong similarities between the objects. Another technique which is used in this paper is fuzzy c-means. FCM algorithm is one of the popular fuzzy clustering algorithms which are classified as constrained soft clustering algorithm. FCM algorithm based on the concept of fuzzy $\mathrm{C}$ partition, which was introduced by various researcher in this field Ruspini [25], developed by Dunn [26] and generalized by Bezdek [27]. A soft clustering algorithm finds a soft partition of a given data set by which an element in the data set may partially belong to multiple clusters. Besides, there is a constriction on the function that the membership degree of a point in all the clusters adds up to 1. The researchers in this field have used SOM or HSOM or FCM separately as one of the tool for the image segmentation for the analysis of tumor.

\section{Related work:}

The image segmentation is the separation of the image into regions of similar feature. In a large number of image processing applications the aim is to extract important features from the image. By using this we can get the description, interpretation or understanding of the image. The segmentation of tumor from images is an important but time-consuming task performed by medical experts. It is very difficult for doctors to see the image again and again, when there is some type of doubt that whether the tumor is present or not in the image (like brain or breast). Several authors suggested various algorithms and methods for detecting tumor automatically in image (Tobias Christian Cahoon et.al (2000); Maria-Luiza Antonie et. al (2001); S. SAHEB BASHA et. al (2005 - 2009); S. Murugavalli et. al (2007); Nahla Ibraheem Jabbar et. al (2008)).

Maria-Luiza Antonie et. al (2001) [13] presented two methods for tumor classification in mammograms. One system exploited the use of neural networks using back-propagation and the second one was built employing association rule mining with constraint form. They segment the image into regions and these regions of interest use single link region growing algorithm. And the features of each region are used as the attributes of the image.

A new approach is presented by S.SAHEB BASHA et. al (2005 - 2009) [15] to identify the existence of breast cancer mass in mammograms. The proposed work utilizes morphological operators for segmentation and fuzzy c- means clustering for clear identification of clusters. The morphological operations and FCM is a new approach, using this they successfully detected the breast cancer masses in mammograms. The results indicate that this system can facilitate the doctor to detect breast cancer in the early stage of diagnosis process. The proposed technique shows better results.

Nahla Ibraheem Jabbar and Monica Mehrotra (2008) [10] used a fuzzy kohonen neural network for medical image segmentation. They analyses the tumor by extraction of the features 
International Journal of Computer Science \& Information Technology (IJCSIT), Vol 3, No 1, Feb 2011

(area, entropy, means and standard deviation).These measurements gives a description for a tumor.

S. Murugavalli et. al (2007) [21], implements a neuro-fuzzy segmentation process of the MRI data to detect various tissues like white matter, gray matter, csf and tumor. A neuro fuzzy based segmentation process is used to detect brain tumor was implemented and they studied the performance of the MRI image in terms of weight vector, execution time and tumor pixels detected and compared the results with the existing ones. A layer by layer abstraction level with fuzzy clustering technique was implemented to detect various tissues like white matter, gray matter, csf and tumor. They achieved a higher value of detected tumor pixels than any other segmentation techniques, also achieved the weight vector value for the neuro fuzzy is $(6 \times 6)$ with the additional input features. The weight vector value, the number of tumor cells and the execution time will also be studied with different distance classifier technique. The change of growth rate of the tumor of the same patient analyze may also be undertaken in this paper.

Tobias Christian Cahoon et.al (2000) [18] describe the use of segmentation with Fuzzy models and classification by the crisp k-nearest neighbor algorithm for assisting breast cancer detection in digital mammograms. Their research utilizes images from the DDSM. They show the supervised and unsupervised methods of segmentation. When only intensity is used as the discriminating features, the k-nn and fuzzy c-means methods will have high misclassification rates in digital mammograms. Adding window means and standard deviations to the feature visually improves segmentations produced by the k-nn rule. While their results are encouraging, other methods are needed to detect smaller pathologies such as microcalcifications.

Sameer Singh and Reem Al-Mansoori [1] find that the fuzzy enhancement methods coupled with either gradient based or fuzzy segmentation techniques are very useful in sketching tumor boundaries in non-dense breasts. Its main aim is to accurately determine regions of interest and to identify and classify tumors as benign or malign, they used information on the textural, shape and spectral characteristics of mammograms.

T.J. Jose et. al (2009) [31] propose a new hybrid approach to content-based image retrieval. In this method a two step approach is used. For clustering the images they use SOM with respect to their basic characteristics in the first step. In the second step, the GA based search will made on a subset of images which were having some basic characteristics of the input query image. This approach was applied to a database of high resolution mammogram images and shows that this method thoroughly improves the retrieval correctness over the single feature vector approach. The results show that the system using GA retrieval doubled the doctors' diagnostic accuracy. As compared to the single stage methods this method is faster and has higher retrieval accuracy.

For MRI brain image segmentation Jianhua Xuan et. al (1995) [6] presents a method on combining the region growing and edge detection methods. Starting with a simple region growing algorithm which produces an over segmented image, they apply a sophisticated region merging method which is capable of handling complex image structures. Integrate edge information to verify and to correct region boundaries. This method is reliable and efficient for MR brain image segmentation.

Luiza Antonie [14] developed a project that performs classification and segment the tumor from the images. He had no extra information that could be very useful in detecting some patterns in developing a certain disease. In this system, the images as well as some patient information are important. Overall, the system performed well, but there is still a lot to improve.

Túlio César Soares dos Santos André et. al [16] develop a digitized mammograms' feature extraction approach using Kohonen's Self-Organizing Maps (SOM). Once developed, 
International Journal of Computer Science \& Information Technology (IJCSIT), Vol 3, No 1, Feb 2011

the SOM network will be used as the first processing stage in a breast cancer computer aided diagnosis (CAD) system. Its role will be to offer segmented data as input to a second stage dedicated to the diagnosis task, which will be implemented via a multi layer perceptron (MLP) trained by the backpropagation algorithm.

\section{Self Organization Map}

Professor Teuvo Kohonen developed the most popular neural network model, the Self Organizing Map (SOM). A self-organizing map (SOM) or self-organizing feature map (SOFM) is a type of artificial neural network that is trained using unsupervised learning to produce a low-dimensional (typically two-dimensional), discretized representation of the input space of the training samples, called a map. Self Organizing Map (SOM) [17] is a member of the CLNNs. When implementing vector quantization using neural network [28-30] this can be one of the greatest choice. SOMs operate in two modes: training and mapping, Training is a competitive process, also called vector quantization. Mapping automatically classifies a new input vector. To extract information from complex medical images, segmentation is an important process. Segmentation has wide application in medical field $[19,20,22]$. The main purpose of the image segmentation is to divide the image into mutually exclusive and exhausted regions. The partition of these regions is such that each region of interest is spatially contiguous and the pixels within the region are homogeneous with respect to a predefined criterion. The SOM [8,9] is a tool for statistical analysis and visualization, for the development of complex applications. Self organizing maps uses a neighbourhood function which are different other than artificial neural networks, to preserve the topological properties of the input space. The arrangement of nodes is a hexagonal or rectangular grid. The mapping in selforganizing map is from higher to lower dimensional input space. To find the node with the closest weight vector, first place a vector taken from data space and second to assign the map coordinates of the node to the vector. The training uses competitive learning. The Euclidean distance to all weight vectors are calculated when a training data is fed to the network. The neurons with weight vectors which are most alike to the input are called as the best matching unit. In the lattice of self organizing map the weights of the best matching unit and the neurons which are close to it are adjusted towards the input vector. From the best matching unit the magnitude of the change decreases with time and distance. The update formula for a neuron with weight vector $\mathrm{Wv}(\mathrm{t})$ is

$$
W v(t+1)=W v(t)+\Theta(v, t) a(t) D((t)-W v(t))
$$

where $\alpha(\mathrm{t})$ is a monotonically decreasing learning coefficient and $\mathrm{D}(\mathrm{t})$ is the input vector. The neighborhood function $\Theta(\mathrm{v}, \mathrm{t})$ depends on the lattice distance, and this lattice distance is between the best matching unit and the neuron v. For all neurons which are close to the best matching unit is one and for others it is zero. The neighborhood function shrinks with time, in spite of the functional form. At the beginning, the self organizing map takes place on the whole scale when the neighborhood is big and the weights are congregated to local estimates, when the neighborhood shrunk to a couple of neurons. For each input vector the process is repeated.

The significance of self organizing map is mainly due to the similarity between the competitive learning process, which is used in the method of SOM and vector quantization. In the segmented image the main drawback of the self organizing map is that the number of neural units in the competitive layer needs to be equal to the desired number of regions. It is not possible to determine a priory the correct number of regions $M$ in the segmented image. This is the main limitation of the conventional SOM for image segmentation. 


\section{Feature extraction}

The purpose of feature extraction is to reduce the original data set by measuring certain properties or features that distinguish one input pattern from another pattern. The extracted feature gives the characteristics of the input by considering the description of the properties of the image to the classifier into a feature space. In this work we calculate three textural features namely contrast, energy and entropy based on the gray level co-occurrence matrices (GLCM). In spite of these we calculate some other features like mean, inverse difference moment and standard deviation. Gray Level Cooccurrence Matrix (GLCM) features are used to distinguish between normal and abnormal image. Five co-occurrence matrices are constructed in four spatial orientations horizontal, right diagonal, vertical and left diagonal $\left(0^{\circ}, 45^{\circ}, 90^{\circ}\right.$, and $\left.135^{\circ}\right)$.

$\operatorname{GLCM}\left\{\mathrm{P}_{(\mathrm{d}, \theta)}(\mathrm{i}, \mathrm{j})\right\}$ represents the probability of occurrence of the pair levels $(\mathrm{i}, \mathrm{j})$ separated by a given distance $d$ at angle $\theta$. In this paper we are considering value of $\theta=0^{\circ}$ and distance $(d)=1$. The commonly used unit pixel distances and the angles are $0^{\circ}, 45^{\circ}, 90^{\circ}$ and $135^{\circ}$. A detailed algorithm of calculation of GLCM $\left\{\mathrm{P}_{(\mathrm{d}, \theta)}(\mathrm{i}, \mathrm{j})\right\}$ has been given in [2]. The features are calculated using the formulae given below.

\section{Contrast:}

$$
S_{c}=\sum_{i=0}^{n} \sum_{j=0}^{m}(i-j) p(i, j)
$$

\section{Entropy:}

$$
S_{e}=-\sum_{i=0}^{n} p(i, j) \log \{p(i, j)\}
$$

\section{Energy:}

$$
S_{e n}=\sum_{i=0}^{n} \sum_{j=0}^{m} p^{2}[i][j]
$$

Mean:

$$
S_{m}=\frac{1}{m \times n}\left\{\sum_{i=0}^{n} \sum_{j=0}^{m}(i p[i, j]+j p[i, j])\right\}
$$

\section{Inverse Difference Moment:}

$$
S_{i d m}=\sum_{i=0}^{n} \sum_{j=0}^{m} p[I, j] /[I-j] \quad \quad \mathrm{i} \neq \mathrm{j}
$$

\section{Standard Deviation:}

$$
S_{d}=\sqrt{\left(\left\{\frac{1}{(m-1)(n-1)}\right\} \sum_{i=0}^{n} \sum_{j=0}^{m}\left(p[i][j]-S_{m}\right)^{2}\right)}
$$

Each set of features are individually normalized to the range of 0 to 255 . These features work well especially for mammograms. 
International Journal of Computer Science \& Information Technology (IJCSIT), Vol 3, No 1, Feb 2011

\section{Implementation of SOM and Fuzzy C-Means}

\subsection{SOM Implementation:}

A self organizing map is an unsupervised clustering property, consists of components called nodes or neurons. This clustering property classifies high dimensional data sets into clusters of similar features. Each node contains a corresponding weight vector of same dimension.

On every step of the learning process a random vector is chosen from the initial data set and then the best-matching (the most similar to it) neuron coefficient vector is identified. Select the winner which is most similar to the input vector [24]. The distance between the vectors usually measured in the Euclidean metric and is given by

$$
\left\|x-W_{c}\right\|=\min _{i}\left\{\left[x-W_{i}\right]\right\}
$$

Where, $\mathrm{x}$ is the neuron, $\mathrm{W}_{\mathbf{C}}$ is the winning neuron vector and $\mathrm{W}_{\mathrm{i}}$ is the weight vector. The modified weight vector coefficients can be calculated by $W_{i}(t+1)=W_{i}(t)+h_{c i}(t) \times\{x(t)-W(t)\}$.

Where $\mathrm{t}$ is the epoch number (discrete-time index), $\mathrm{x}(\mathrm{t})$ is the vector and is obtained by selecting a sample randomly for iteration $t$. The function $h_{\mathrm{ci}}(\mathrm{t})$ is called neighborhood function and it represents a non-increasing function of time and the distance between the winning neuron and its neighbors on the grid. The function $\mathrm{h}_{\mathrm{ci}}(\mathrm{t})$ divides into of two parts, first part is the proper distance function and second is the learning rate function. And this can be defined as $h(t)=h\left(\left\|r_{c}-r_{i}\right\|\right) \times a(t)$ where, $\mathrm{r}_{\mathrm{c}}$ is the position of winning neuron and the position of its neighbor is $r_{i}$. The result of neighborhood function $h(t)$ is an initial cluster center (centroids) for fuzzy c means algorithms.

\section{Stepping through the algorithm}

1. Randomize the map's nodes' weight vectors.

2. Grab an input vector.

3. Traverse each node in the map

i. Find similarity between the input vector and the weight vector, using Euclidean distance formula.

ii. Track the node which shows the smallest distance (this node is called as best matching unit).

4. Update the nodes in the neighborhood of BMU by pulling them closer to the input vector.

$$
W_{i}(t+1)=W_{i}(t)+h_{c i}(t) \times\{x(t)-W(t)\}
$$

where $\mathrm{W}_{\mathrm{i}}$ is current weight vector.

5. Increment $t$ and repeat from step 2 while $t<\lambda$, where $t$ is current iteration and $\lambda$ is limit on time iteration.

\subsection{FCM Implementation:}

Fuzzy c-means is a clustering method which allows to find cluster centers that minimize dissimilarity functions [3,4]. In order to accommodate the fuzzy partitioning technique, the membership matrix $(\mathrm{U})$ is randomly initialized as: 
International Journal of Computer Science \& Information Technology (IJCSIT), Vol 3, No 1, Feb 2011

$$
\sum_{i=1}^{c} U_{i j}=1, \forall j=1, \ldots \ldots . n
$$

where $i$ and $j$ are the number of cluster and image data points respectively. The dissimilarity function can be computed as:

$$
J\left(U, c_{1}, c_{2}, \ldots . c_{c}\right)=\sum_{i=1}^{c} J_{i}=\sum_{i=1}^{c} \sum_{j=1}^{n} u_{i j}{ }^{m} d_{i j}{ }^{2}
$$

where $u_{i j}$ lies between 0 and $1 . d_{i j}$ is the Euclidian distance between $i_{t h}$ centroid $\left(c_{i}\right)$ and $j_{t h}$ data point. The weighting exponent is $m$ and its value is greater than one. The minimum of dissimilarity function can be computed as:

$$
u_{i j}=1 / \sum_{k=1}^{c}\left(d_{i j} / d_{k j}\right)^{2 /(m-1)}
$$

where, $\mathrm{d}_{\mathrm{ij}}=\left\|\mathrm{x}_{\mathrm{i}}-\mathrm{c}_{\mathrm{j}}\right\|, \mathrm{d}_{\mathrm{kj}}=\left\|\mathrm{x}_{\mathrm{i}}-\mathrm{c}_{\mathrm{k}}\right\|, x_{i}$ is the $\mathrm{i}$ th of d-dimensional data, $c_{j}$ is the d-dimension center of the cluster and $\|*\|$ is any norm expressing the similarity between any measured data and center. This iteration will stop when $\left.\operatorname{Max}_{\mathrm{ij}}\left|\mathrm{u}_{\mathrm{ij}}{ }^{(\mathrm{k}+1)}-\mathrm{u}_{\mathrm{ij}}{ }^{(\mathrm{k})}\right|\right\}<\varepsilon$, where $\varepsilon$ is a termination criterion between 0 and 1 , whereas $k$ are the iteration steps.

\section{Fuzzy c-means algorithm (FCM):}

Step1:

Initialize the membership matrix, $\mathrm{U}=\left[\mathrm{u}_{\mathrm{ij}}\right]$.

Step 2:

At $\mathrm{k}_{\mathrm{th}}$ number of iteration:

Calculate the center vectors $\mathrm{c}_{\mathrm{i}}$ with $\mathrm{u}_{\mathrm{ij}}$

$$
c_{i}=\sum_{i=1}^{n} u_{i j}{ }^{m} \times x_{i} / \sum_{j=1}^{n} u_{i j}{ }^{m}
$$

Step3:

Update the membership matrix $\mathrm{U}$ for the $\mathrm{k}_{\mathrm{th}}$ steps and $(\mathrm{k}+1)_{\mathrm{th}}$ step.

$$
u_{i j}=1 / \sum_{k=1}^{c}\left(d_{i j} / d_{k j}\right)^{2 /(m-1)}
$$

where $d_{i j}=x_{j}-c_{i}$

Steps4:

If $\|\mathrm{U}(\mathrm{k}+1)-\mathrm{U}(\mathrm{k})\|<\varepsilon$ then STOP; otherwise return to step2.

\section{Result}

The result of the implementation of the neuro fuzzy region classification process is discussed in this section. For analyzing medical images, execution time is one of the main constraints of any computer aided analysis. We calculate the number of tumor pixels which are affected by tumor cells in the medical image. In this paper we input fixed size and noiseless mammogram images $($ size $=256 \times 256)$ only. The images used are $256 \times 256$ gray level images with intensity value ranges from (0 to 255). In this paper we calculated the tumor properties like location, energy, 
International Journal of Computer Science \& Information Technology (IJCSIT), Vol 3, No 1, Feb 2011

entropy, IDM, contrast, mean, standard deviation and image properties like type of the region, average gray value of region, area of that region, centroid of the region.

The execution time of this technique found to be 50 to 65 seconds and detected tumor pixels are approximately 588. Table- 1 and Table- 2 shows the input of abnormal and normal mammography image and their tumor properties respectively. Some screenshots of the GUI are shown below by extracting tumor and their properties of normal and abnormal mammography images.

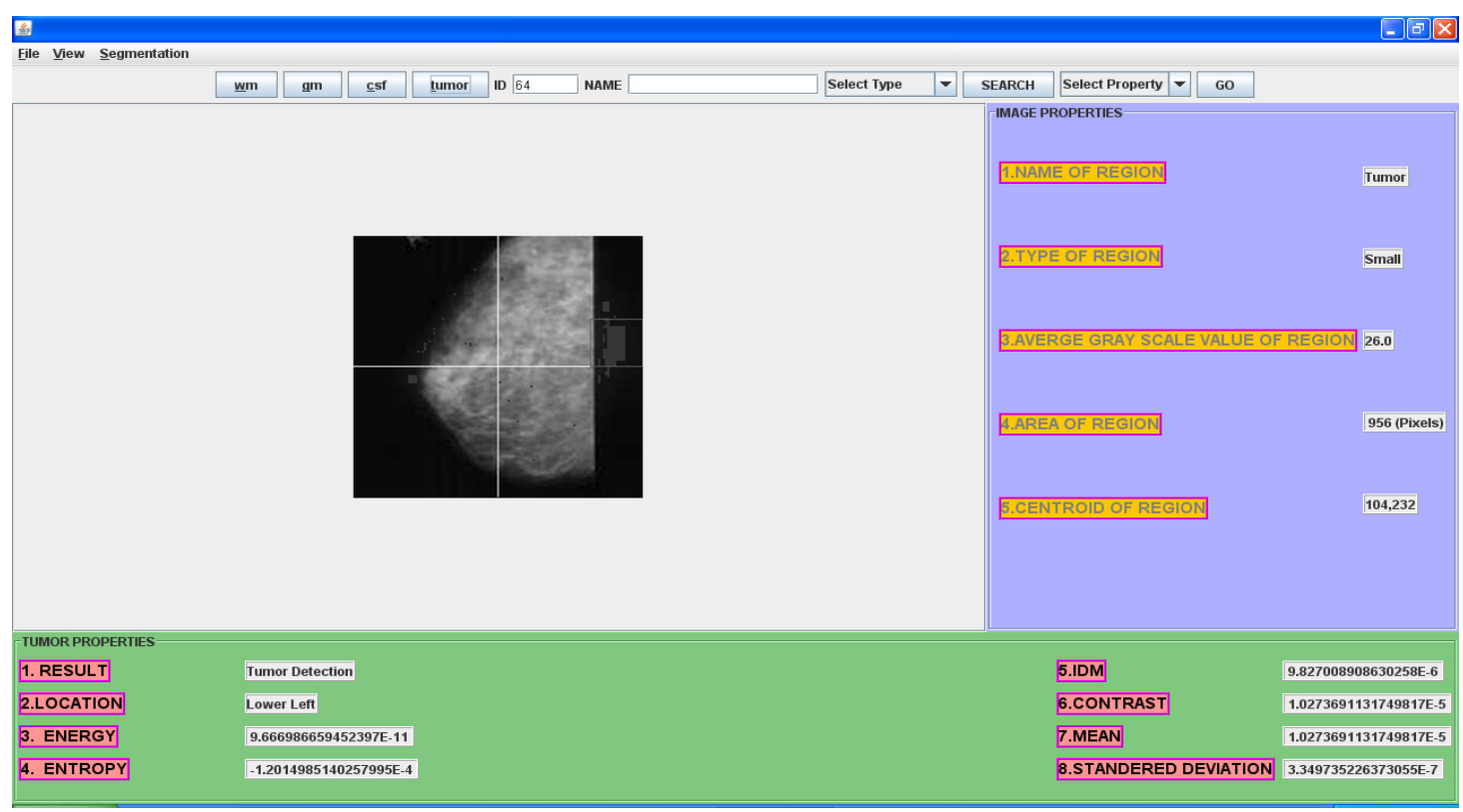

Figure1: Snapshot of GUI of Abnormal Mammography Image and its properties.

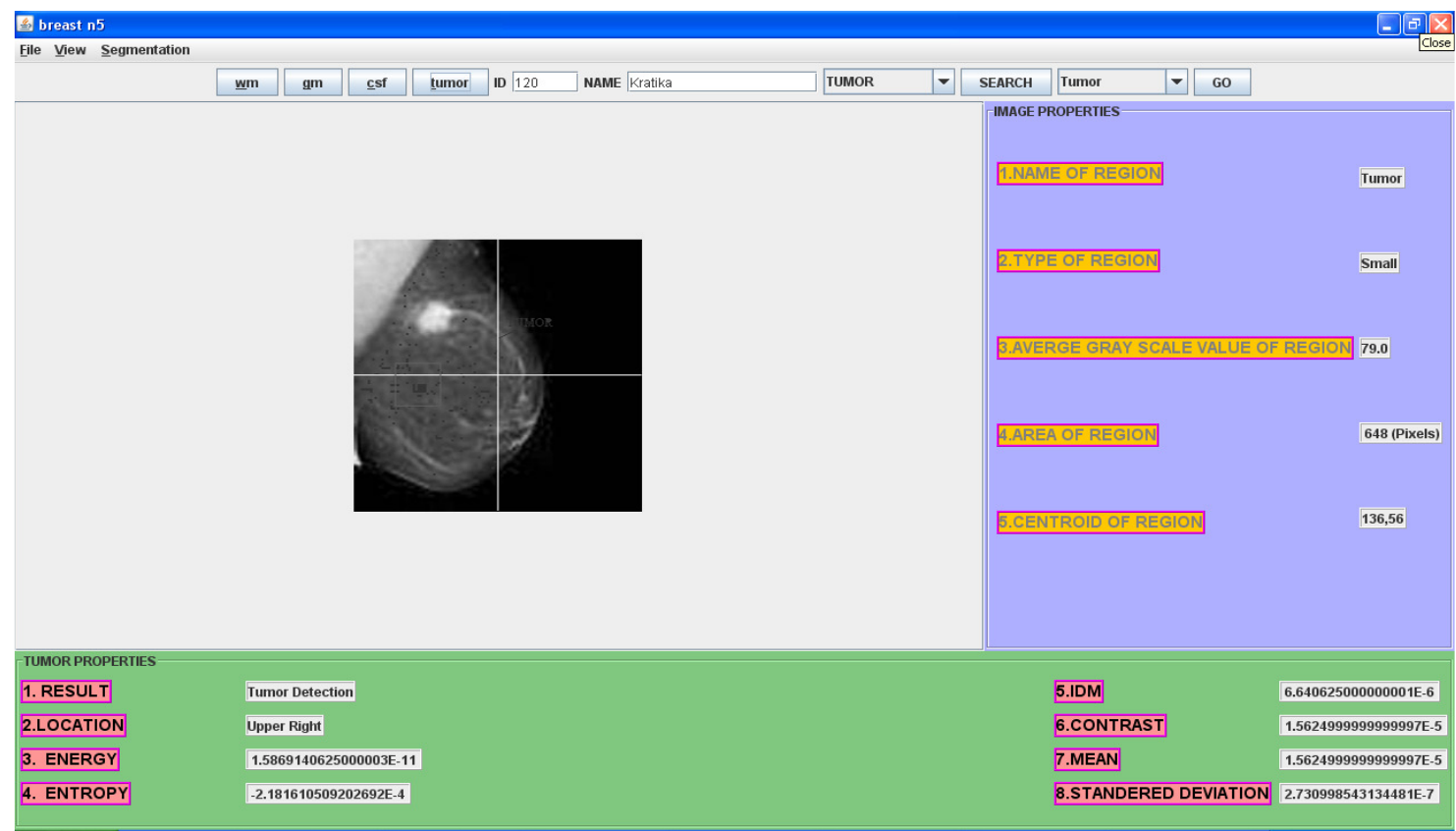

Figure2: Snapshot of GUI of Abnormal Mammography Image and its properties. 
International Journal of Computer Science \& Information Technology (IJCSIT), Vol 3, No 1, Feb 2011

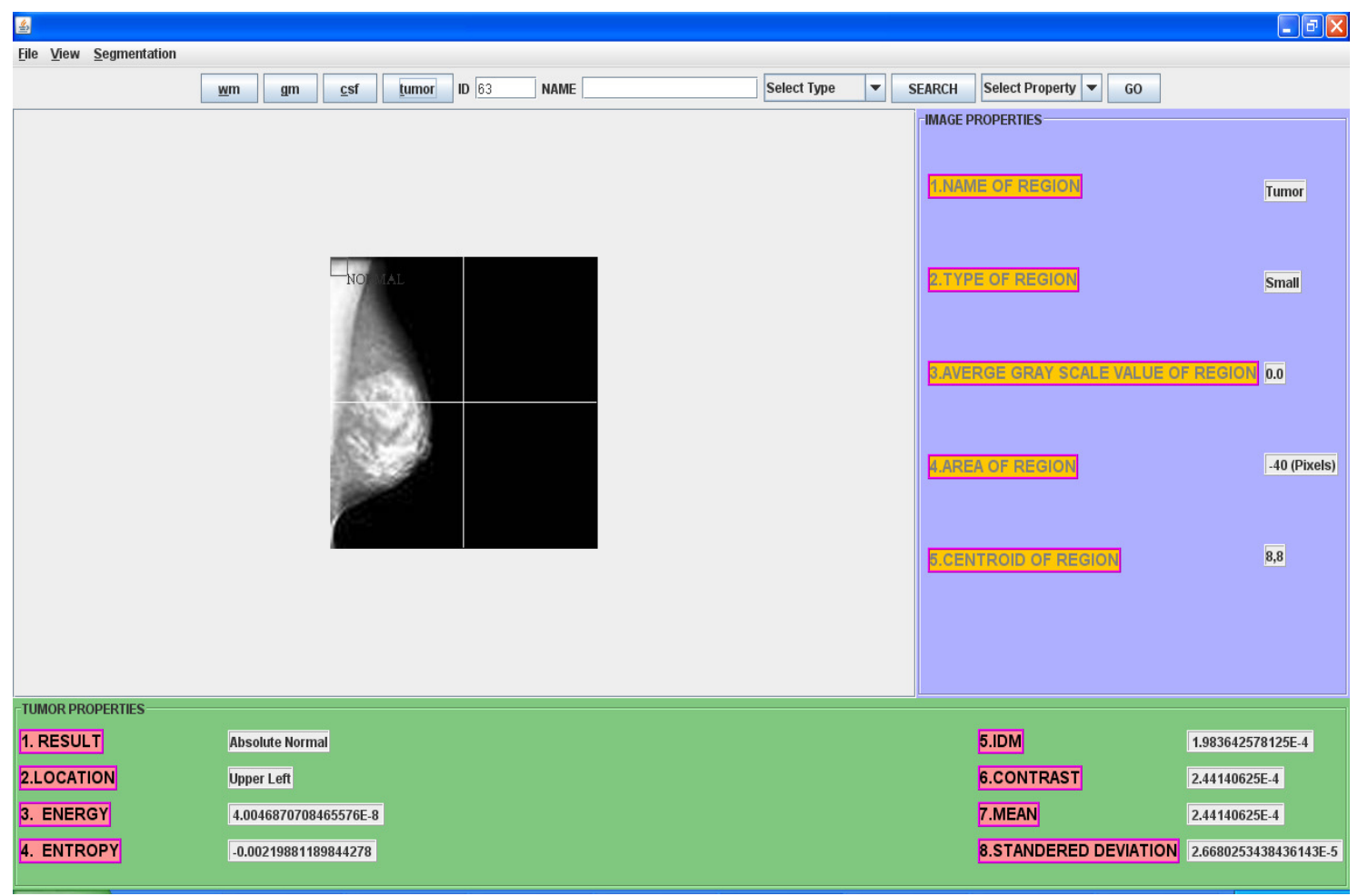

Figure3: Snapshot of GUI of Absolute normal Mammography image and its properties.

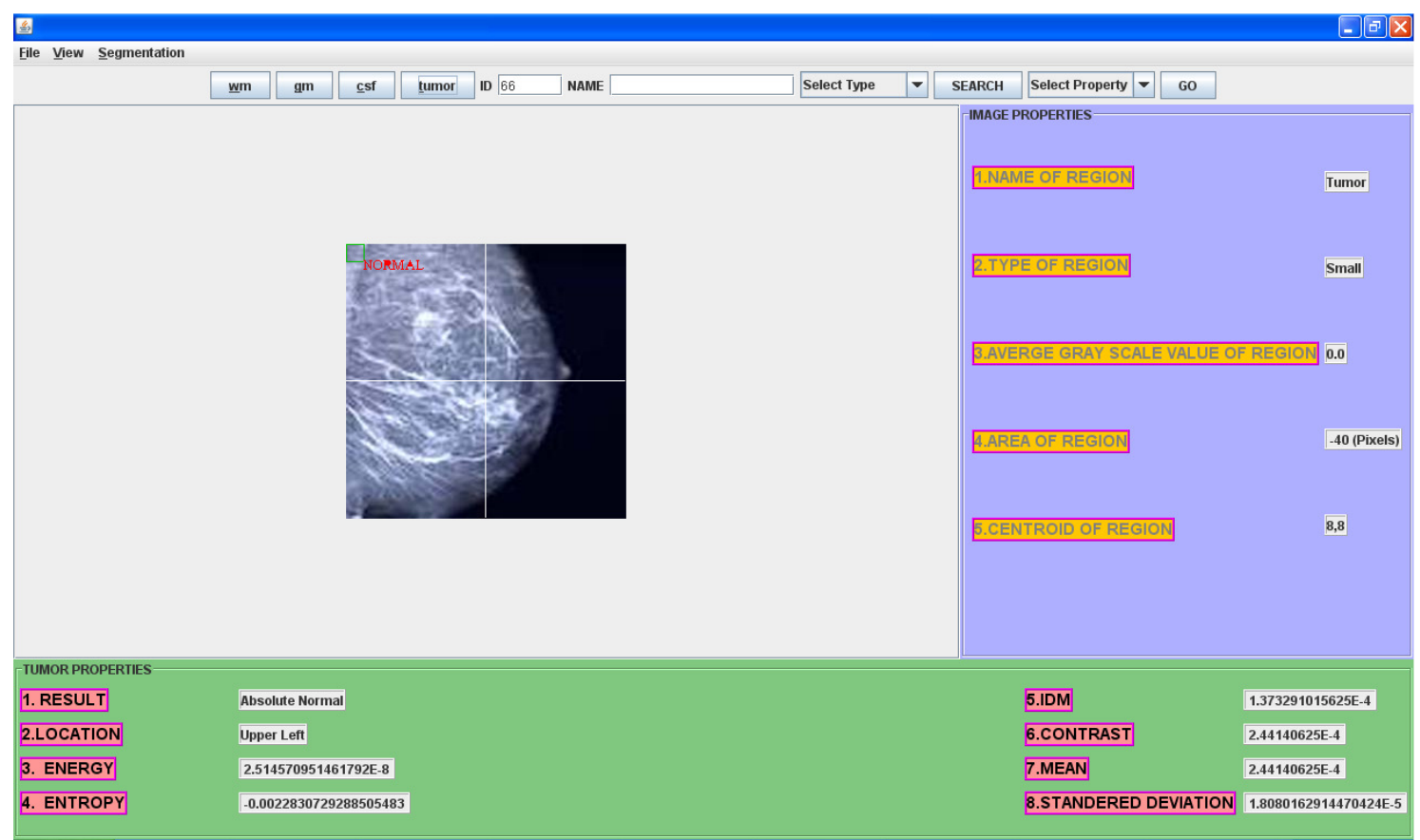

Figure4: Snapshot of GUI of Absolute normal Mammography Image and its properties. 
International Journal of Computer Science \& Information Technology (IJCSIT), Vol 3, No 1, Feb 2011

\begin{tabular}{|c|c|c|c|c|}
\hline \multirow[t]{8}{*}{ e(1) } & \multirow{2}{*}{$\begin{array}{c}\begin{array}{c}\text { Image } \\
\text { Properties }\end{array} \\
\text { Name of region } \\
\end{array}$} & \multirow{2}{*}{$\begin{array}{l}\text { Tumor } \\
\text { Tumor } \\
\end{array}$} & \multicolumn{2}{|r|}{$\begin{array}{c}\text { Tumor Properties } \\
\text { (Abnormal) }\end{array}$} \\
\hline & & & Location & Lower left \\
\hline & Type of region & Small & Energy & $9.666986659452397 \mathrm{E}-11$ \\
\hline & $\begin{array}{l}\text { Average gray } \\
\text { scale value of } \\
\text { region }\end{array}$ & 26.0 & Entropy & $-1.2014985140257995 \mathrm{E}-4$ \\
\hline & $\begin{array}{l}\text { Area of region } \\
\text { (pixels) }\end{array}$ & 956 & IDM & $9.827008908630258 \mathrm{E}-6$ \\
\hline & $\begin{array}{l}\text { Centroid of } \\
\text { region }\end{array}$ & 104,232 & Contrast & $1.0273691131749817 \mathrm{E}-5$ \\
\hline & & & Mean & $1.0273691131749817 \mathrm{E}-5$ \\
\hline & & & $\begin{array}{l}\text { Standard } \\
\text { deviation }\end{array}$ & $3.349735226373055 \mathrm{E}-7$ \\
\hline Image (2) & $\begin{array}{c}\text { Image } \\
\text { Properties }\end{array}$ & Tumor & \multicolumn{2}{|c|}{$\begin{array}{c}\text { Tumor Properties } \\
\text { (Abnormal) }\end{array}$} \\
\hline & Name of region & Tumor & Location & Upper Right \\
\hline & Type of region & Small & Energy & $1.5869140625000003 \mathrm{E}-11$ \\
\hline & $\begin{array}{l}\text { Average gray } \\
\text { scale value of } \\
\text { region }\end{array}$ & 79.0 & Entropy & $-2.181610509202692 \mathrm{E}-4$ \\
\hline & $\begin{array}{l}\text { Area of region } \\
\text { (pixels) }\end{array}$ & 648 & IDM & $6.640625000000001 \mathrm{E}-6$ \\
\hline & $\begin{array}{l}\text { Centroid of } \\
\text { region }\end{array}$ & 136,56 & Contrast & 1.5624999999999997E-5 \\
\hline & & & Mean & $1.5624999999999997 \mathrm{E}-5$ \\
\hline & & & $\begin{array}{l}\text { Standard } \\
\text { deviation }\end{array}$ & $2.730998543134481 \mathrm{E}-7$ \\
\hline
\end{tabular}

Table 1:- Shows the input abnormal mammography images and their properties.

\begin{tabular}{|c|c|c|c|c|}
\hline \multirow[t]{8}{*}{ Image (3) } & $\begin{array}{c}\text { Image } \\
\text { Properties }\end{array}$ & Tumor & \multicolumn{2}{|c|}{$\begin{array}{l}\text { Tumor Properties } \\
\text { (Absolute Normal) }\end{array}$} \\
\hline & Name of region & Tumor & Location & Upper left \\
\hline & Type of region & Small & Energy & $4.0046870708465576 \mathrm{E}-8$ \\
\hline & $\begin{array}{l}\text { Average gray } \\
\text { scale value of } \\
\text { region }\end{array}$ & 0,0 & Entropy & -0.0021988189844278 \\
\hline & $\begin{array}{l}\text { Area of region } \\
\text { (pixels) }\end{array}$ & -40 & IDM & $1.983642578125 \mathrm{E}-4$ \\
\hline & $\begin{array}{l}\text { Centroid of } \\
\text { region }\end{array}$ & 8,8 & Contrast & $2.44140625 \mathrm{E}-4$ \\
\hline & & & Mean & $2.44140625 \mathrm{E}-4$ \\
\hline & & & $\begin{array}{l}\text { Standard } \\
\text { deviation }\end{array}$ & $2.6680253438436143 \mathrm{E}-5$ \\
\hline Image (4) & $\begin{array}{c}\text { Image } \\
\text { Properties }\end{array}$ & Tumor & \multicolumn{2}{|r|}{$\begin{array}{l}\text { Tumor Properties } \\
\text { (Absolute Normal) }\end{array}$} \\
\hline 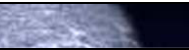 & Name of region & Tumor & Location & Upper left \\
\hline
\end{tabular}


International Journal of Computer Science \& Information Technology (IJCSIT), Vol 3, No 1, Feb 2011

\begin{tabular}{|l|l|l|l|l|}
\hline & Type of region & Small & Energy & $2.514570951461792 \mathrm{E}-8$ \\
\cline { 2 - 5 } & $\begin{array}{l}\text { Average gray } \\
\text { scale value of } \\
\text { region }\end{array}$ & 0,0 & Entropy & -0.0022830729288505483 \\
\cline { 2 - 5 } & $\begin{array}{l}\text { Area of region } \\
\text { (pixels) }\end{array}$ & -40 & IDM & $1.373291015625 \mathrm{E}-4$ \\
\cline { 2 - 5 } & $\begin{array}{l}\text { Centroid of } \\
\text { region }\end{array}$ & 8,8 & Contrast & $2.44140625 \mathrm{E}-4$ \\
\hline & & Mean & $2.44140625 \mathrm{E}-4$ \\
\hline & $\begin{array}{l}\text { Standard } \\
\text { deviation }\end{array}$ & $1.8080162914470424 \mathrm{E}-5$ \\
\hline
\end{tabular}

Table 2:- Shows the input absolute normal mammography images and their properties.

\section{Conclusion}

A neuro fuzzy based segmentation process is used to characterize the tumor was implemented. We studied the performance of the Digital Mammography images in terms of execution time and tumor pixels detected. Fuzzy clustering technique was implemented to detect the abnormality of mammography image and finding some statistical properties. We have achieved a higher value of detected tumor pixels. Medical images generally contain unknown noise and considerable uncertainty, and therefore clinically acceptable segmentation (region classification) performance is difficult to achieve. In this paper we have input fixed size and noiseless mammogram image $($ size $=256 \times 256)$ only. We can find whether there is some abnormality present or not in the input mammography image. We have determined a limit for detected abnormal pixels, if number of abnormal pixels are less than this range, than no tumor, else tumor detected. In future the study is continuing for segmentation of variable size and noisy mammogram images, detecting the type of tumor and decreasing its execution time.

\section{References}

[1] "IDENTIFICATION OF REGIONS OF INTEREST IN DIGITAL MAMMOGRAMS", by Sameer Singh and Reem Al-Mansoori.

[2] "The handbook of pattern recognition and computer vision", by C.H.Chen, L.F.Pau, P.S.P.Wang, 2nd edition, World scientific publishing company, 1998.

[3] "A Parallel Fuzzy C-Mean Algorithm for Image segmentation”, by S. Rahmi, M. Zargham, A. Thakre and D. Chhillar, NAFIPS 04, IEEE Annual meeting, 1: 234-237, (2004).

[4] Hung, M.C. and D.L. yang, 2001. An Efficient Fuzzy C means Clustering Algorithm, Data mining, ICDM 2001, Proceedings IEEE International conference on pp: 225-232.

[5] K.Woods, C. Doss, K.W. Bowyer, J.L. Solka, C.E. Priebe, and W.P. Kegelmeyer, "Comparative evaluation of pattern recognition techniques for detection of microcalcifications in mammography", International Journal of Pattern Recognition and Artificial Intelligence 7 (6), 1417-1436, (December 1993). 
International Journal of Computer Science \& Information Technology (IJCSIT), Vol 3, No 1, Feb 2011 [6] Jianhua Xuan, Tiilay Adali, Yue Wang, "SEGMENTATION OF MAGNETIC RESONANCE BRAIN IMAGE: INTEGRATING REGION GROWING AND EDGE DETECTION”, IEEE 1995.

[7] T. M. Freer and M. J. Ulissey, "Screening mammography with computer-aided detection: prospective study of 12,860 patients in a community breast center," Radiology 220, pp. 781-786, 2001.

[8] Juha Vesanto and Esa Alhoniemi. Clustering of the Self-Organizing Map. IEEE Transactions on Neural Networks, 11(2):586-600, March 2000.

[9] "Distance matrix based clustering of the self organizing map", by Juha Vesanto and Mika Sulkava. International Conference on Artificial Neural Networks-ICANN 2002, Madrid, Spain, August 28-30, pages $951-956$.

[10] "Application of Fuzzy Neural Network for Image Tumor Description", by Nahla Ibraheem Jabbar, and Monica Mehrotra, proceedings of world academy of science, engineering and technology volume 34 october 2008 ISSN 2070-3740.

[11] G. Lemaur, K. Drouiche, J. DeConinck, "Highly regular wavelets for the detection of clustered microcalcifications in mammograms". IEEE Trans. Medi. Imag., 22: 393-401. DOI: 10.1109/TMI.2003.809580.

[12] "A CAD System for the automatic detection of clustered Microcalcification in digitized mammogram films", by Yu Songyang, and Guan Ling, IEEE Trans. Med. Imag., 19: 115-126. DOI: 10.1109/42.836371, 2000.

[13] "Application of Data Mining Techniques for Medical Image Classification", by Maria-Luiza Antonie, Osmar R. Zaiane, Alexandru Coman, proceedings of the International Workshop on Multimedia Data Mining (MDM/KDD'2001), in conjunction with ACM SIGKDD conference, San Grancisco, USA, August 26, 2001.

[14] Luiza Antonie, project on "Automated Segmentation and Classification of Brain Magnetic Resonance Imaging".

[15] "AUTOMATIC DETECTION OF BREAST CANCER MASS IN MAMMOGRAMS USING MORPHOLOGICAL OPERATORS AND FUZZY C -MEANS CLUSTERING”, by S.SAHEB BASHA, DR.K.SATYA PRASAD, Journal of Theoretical and Applied Information Technology, 2005 2009 JATIT.

[16] "Segmentation of Digitized Mammograms Using Self-Organizing Maps in a Breast Cancer Computer Aided Diagnosis System", by Túlio César Soares dos Santos André, Antônio Carlos Roque da Silva Filho. Proceedings of the VII Brazilian Symposium on Neural Networks (SBRN'02), 0-7695-17099/02 \$17.00 @ 2002 IEEE.

[17] Parra CA, Iftekharuddin K, Kozma R (2003), "Automated Brain Tumor segmentation and pattern recognition using ANN”, Computational Intelligence Robotics and Autonomous Systems.

[18] "Breast cancer detection using image processing techniques", by Tobia Christian Cahoon, Melanie A. Sutton, James C. Bezdek, IEEE 2000.

[19] "Neural Network based segmentation of Magnetic Resonance Images of the Brain", by J Alirezaie, ME Jernigan, C Nahmias, IEEE Trans. Nucl. Sci. 44(2): 194-198, 1997. 
International Journal of Computer Science \& Information Technology (IJCSIT), Vol 3, No 1, Feb 2011 [20] "Survey, image segmentation techniques", by Haralick RM, Shapiro LG, Computer Vision, Graphics Image Process 29: 100-132, 1985.

[21] "An Improved Implementation of Brain Tumor Detection Using Segmentation Based on Neuro Fuzzy Technique", by S. Murugavalli and V. Rajamani, Journal of Computer Science 3 (11): 841-846, ISSN 1549-3636, (2007).

[22] "A review on image segmentation techniques", by Pal NR and Pal SK, Pattern Recognition 26(9): 1277-1294, 1993.

[23] A. S. Feing, Decreased cancer mortality through mammographic screening: results of clinical trials, Radiology, 167: 659-665 (1988).

[24] "A Hierarchical SOFM for analysis of not well separable Clusters of different Feature Density", by S. Schunemann, and B. Michaelies, Symposium on ANN, Bruges (Belgium), pp: 21-23, ESANN'1999.

[25] Ruspini, E., 1970. Numerical methods for fuzzy clustering, Information Sciences, 2: 319-350.

[26] "A fuzzy relative of the ISODATA process and its use in detecting compact, well Separated clusters", by Dunn, J.C., Journal of Cybernetics, 3: 32-51, (1973).

[27] "Cluster validity with fuzzy sets", by J.C. Bezdek, Cybernetics, 3: 58-73, 1974.

[28] "Self-Organization and Associative Memory", by T. Kohonen, 2nd Edition (Springer-Verlag, Berlin, Germany), 1988.

[29] "Segmentation using neural networks for automatic thresholding", by A. Scherf and G. Roberts. in: S. Rogers (ed.), Proc. SPIE Conference on "Applications of Artificial Neural Networks", (Orlando, FL, 1294), pp: 118-124, 1990.

[30] "Constraint satisfaction neural networks for image segmentation", by Lin W., E. Tsao and C. Chen, In: T.Kohonen, K. Mkisara, 0. Simula and J. Kangas (eds.), Artificial Neural Networks (Elsevier Science Publishers), pp: 1087-1090, (1991).

[31] "Neural Network and Genetic Algorithm Based Hybrid Model for content based mammogram image retrieval", by T.J. Jose and P.Mythili, Journal of applied sciences 9(19): 3531-3538, 2009, ISSN $1812-5654$. 